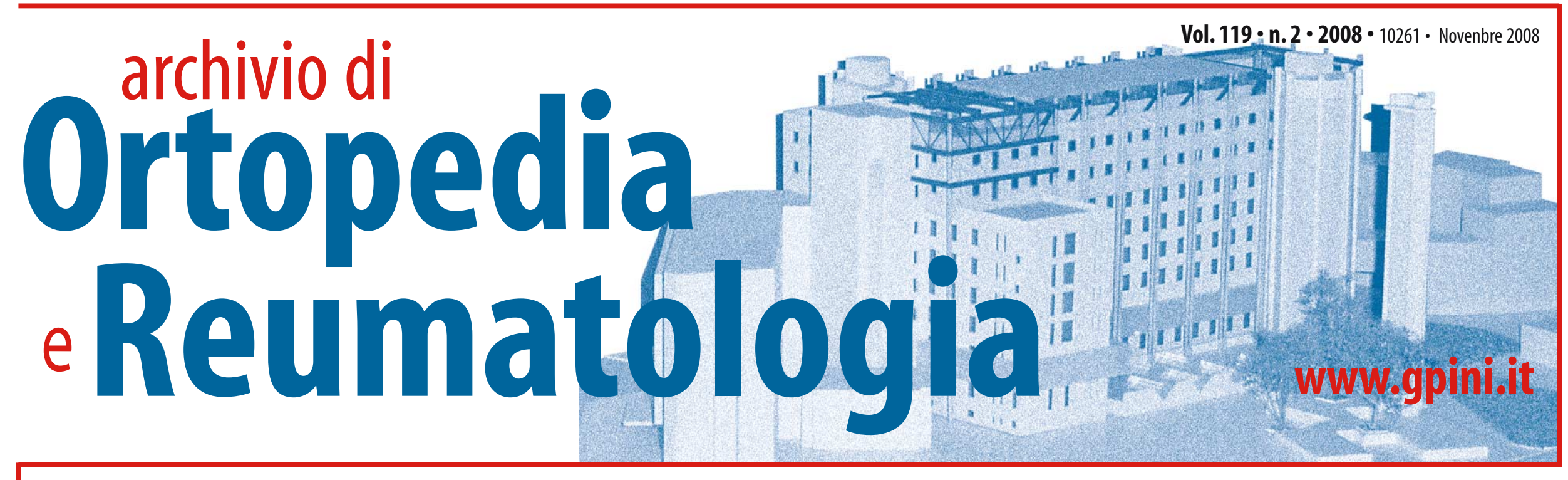

\title{
Le ernie del disco
}

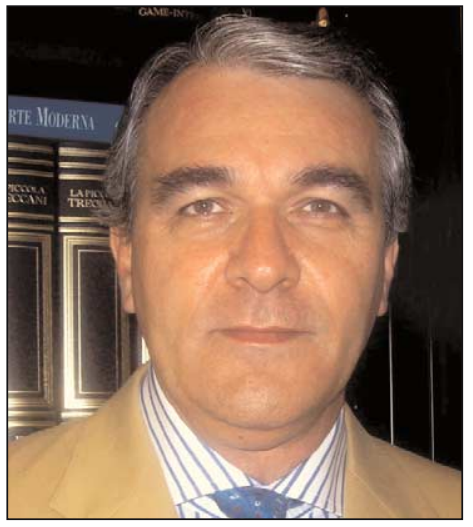

S. Brambilla

0 uesto numero di Archivio di Ortopedia e Reumatologia è dedicacerto, si dirà che non è un argomento originale, che tutto è già stato scritto e riscritto, che...

Tutto vero, come vero è il fatto che il problema riguarda un gran numero di persone, siano essi pazienti oppure tutti coloro che a vario titolo si vedono impegnati nella cura di questi malati; tema perciò "vecchio", ma sempre di grande attualità, probabilmente un po' dimenticato e forse trascurato proprio perché non di moda. Inoltre si tratta di un argomento che vede coinvolte più branche specialistiche, motivo per cui mi sono rivolto ai colleghi neurologi, radiologi, reumatologi, anestesisti-terapisti del dolore, fisiatri, neurochirurghi, oltre che a ortopedici e a una personalità giuridica, proprio per cercare di avere una visione a 360 gradi.

Ovviamente grande risalto è stato dato all'ernia del disco lombare, in quanto si tratta di una patologia estremamente frequente, con alto impatto sociale, terreno di competenza di più operatori sanitari. Ho pensato però di lasciare un po' di spazio anche alla trattazione delle ernie discali cervicali e dorsali, di riscontro decisamente meno frequente, condizioni ancor oggi poco conosciute e per molti aspetti sottovalutate. In particolare, le ernie dorsali o toraciche (circa l'1\% di tutte le ernie del disco) costituiscono un capitolo a parte, per la rarità, la sede e il tipo di trattamento chirurgico specifico che richiedono, bagaglio tecnico e culturale di pochi esperti, per le possibili catastrofiche conseguenze che un trattamento incongruo può provocare.
Le ernie cervicali vengono attualmente riscontrate con maggiore frequenza, grazie alle moderne tecniche di "imaging"; il loro trattamento è riservato a un numero ristretto di esperti, soprattutto se si considera l'aspetto chirurgico. Una importante considerazione è che, nel rispetto di corrette indicazioni, i risultati chirurgici sono sicuramente ottimi e duraturi nel tempo e ciò è dovuto a mio parere, ad alcuni fattori decisivi, quali una tecnica "radicale" collaudata e sostanzialmente sicura e una selezione per certi versi "naturale" dei pazienti, poco propensi all'intervento chirurgico - percepito come difficile e rischioso - i quali si sottopongono quindi con più motivazione e attenzione ai trattamenti conservativi, che risultano spesso risolutivi.

Il segmento lombare del rachide è comunque quello più coinvolto e verso il quale si concentra l'attenzione della maggior parte della popolazione. Il mal di schiena è una condizione estremamente frequente nelle società industrializzate e si ritiene che 1'80\% dei soggetti adult soffra, prima o poi, di almeno un episodio di lombalgia acuta nell'arco della vita.

Nell'85\% dei soggetti affetti da lombalgia la causa del dolore non viene identificata con certezza e quindi non viene formulata una diagnosi precisa e definitiva: si parla pertanto di lombalgia aspecifica. Il mal di schiena è una delle cause più frequenti di richiesta di visita medica o di accesso al Pronto Soccorso, anche se nella stragrande maggioranza dei casi la sintomatologia regredisce nel giro di pochi giorni, spesso senza una terapia specifica; altre volte l'intensità del dolore comporta la perdita di giornate lavorative, con un costo sociale elevato. La degenerazione del disco intervertebrale è una delle principali ragioni di mal di schiena e anche se il reale rapporto fra lombalgia e degenerazione discale non è ancora ben compreso, si stima che più del $90 \%$ dei trattamenti chirurgici sul rachide venga effettuato in conseguenza della degenerazione del disco intervertebrale.

Lavvento delle nuove tecniche di "imaging" ha permesso sicuramente una migliore visualizzazione delle strutture anatomiche vertebrali, ma questo non sempre si accompa- gna a una più chiara e sicura interpretazioni delle immagini. In altre parole, il reale rapporto fra immagini e espressione clinica è tuttora lontano dall'essere completamente compreso e acquisito. Sappiamo che i processi degenerativi sono collegati con l'invecchiamento, ma non sappiamo, per esempio, quale significato abbia il rilevamento, su una radiografia standard, della per dita di altezza di uno o più dischi intervertebrali: essa potrebbe infatti appartenere a un soggetto completamente asintomatico, così come un soggetto sofferente da anni di lombalgia cronica. Neppure la RMN, attraverso la dimostrazione di una diminuzione di intensità di segnale nelle sequenze sagittali T2 pesate ("black disc" degli Autori anglosassoni) riesce a identificare con sicurezza la sede di provenienza del dolore: tale segno esprime sicuramente la perdita di contenuto in acqua del disco, ma non può e non deve essere interpretato come spiegazione certa ed esclusiva della sintomatologia.

A volte la RMN può addirittura ingenerare perplessità o indurre a diagnosi errate, attraverso una sovrastima delle immagini fornite. $\overrightarrow{\mathrm{E}}$ or mai dimostrato che modificazion del contorno del disco intervertebrale (protrusioni, "bulging", ernie) sono presenti in maniera significativa e progressivamente maggiore con il passare degli anni, in RMN di soggetti completamente asintomatici, e che quindi bisogna dare giusto peso al loro riscontro, pe non correre il rischio di "curare le immagini e non i pazienti”.

Negli Stati Uniti vi è stato un vertiginoso aumento di interventi chirurgici sul rachide, spesso ingiustificato e legato alla introduzione di nuove tecniche e nuovi materiali su una base spesso solo commerciale, senza un supporto di evidenz scientifica. Fra il 1996 e il 2001 gli interventi di artrodesi vertebrale sono aumentati del $77 \%$, contro un aumento, nell'identico periodo, del $13 \%$ e $14 \%$ rispettivamente per le protesi d'anca e di ginocchio. Ciò ha contribuito all'aumento della spesa sanitaria sia in maniera diretta (in media 34.000 dollari per ogni intervento di fusione vertebrale esclusi i compensi professionali) sia come "rebound" per il contemporaneo aumento degli insuccessi chirurgici: negli Stati Uniti, in conseguenza di uno stimato "overtreatment" di circa 2-4 volte, vi è il 15\% di casi di "failed back syndrome", rispetto al 5\% dei Paesi europei; circa la metà di questi va incontro successivi trattamenti chirurgici per lombalgia cronica, finendo per assorbire circa i 3/4 dell'intera spesa sanitaria destinata al "back pain"

Il messaggio che intendiamo lanciare è quindi quello di considerare at tentamente il quadro clinico e il soggetto nella sua totalità, per evitare molti interventi (troppi?) inutili o addirittura forieri di situazioni an cora più invalidanti e croniche.

Il mal di schiena ("low back pain") comune o aspecifico rimane comunque un sintomo, non è una diagnosi! Per ernia del disco si intende una patologia ben codificata che si esprime attraverso una sintomatologia chiara, supportata e in accordo con le tecniche di "imaging".

Riferendosi a dati statistici degli anni Ottanta, risulta che in Gran Bretagna vengono eseguiti interven per ernia discale lombare con un frequenza di 10 per 100.000 abitanti, mentre negli Stati Uniti si passa a oltre 100 per 100.000 .

L'indagine ISTAT pubblicata nel
2000 sullo stato di salute dei cittadini italiani ha evidenziato una frequenza di lombosciatalgia pari a $7,3 \%$ per i maschi e $9,3 \%$ per le femmine; i dati dell'Istituto Superiore di Sanità dicono che ogni anno in Italia vengono sottoposti a trattamento chirurgico per ernia del disco lombare circa 30.000 pazienti, con un'incidenza media su scala nazionale pari a 5,1 interventi ogni 10.000 abitanti nel triennio 1999 2001 e con variazioni regionali significative: $6,9 \%$ in Lombardia e 2,5\% in Calabria. Nel 61\% dei casi prevale il sesso maschile, la fascia di età più interessata è quella fra 30 e 50 anni e le procedure chirurgiche sono rappresentate da discectomia (88\%), discectomia percutanea o laser (7\%) e chemonucleolisi (2\%). 'avvento di tecniche cosiddette mini-invasive ha suscitato grande interesse nell'opinione pubblica e ha indotto le persone che soffrono di lombalgia a considerare questi trattamenti con maggiore fiducia e minore ansia e preoccupazione rispetto alle tradizionali soluzioni chirurgiche. Il problema non è però quello di far adattare i pazienti alle tecniche o ancor più alle sole tecniche che conosciamo! Lobiettivo deve essere quello di rispondere alle esigenze di salute dei malati nel modo migliore, con il minor rischio, senza promesse di guarigioni prodigiose $e$ immediate. Siamo stati e siamo tuttora testimoni di soluzioni spacciate per rivoluzionarie attraverso un "battage" pubblicitario spesso incontrollato, senza un valido substrato scientifico di supporto, così che si assiste comunque a un "overtreatment", anche se ovviamente mini-invasivo. Alcune di queste metodiche sono cadute in disuso, altre conservano ancora una loro continua a pag. 5 


\title{
Ruolo dell'elettroneurofisiologia nelle radicolopatie
}

\author{
M. Loiero
}

U. O. di Neurologia, Istituto Ortopedico G. Pini, Milano

\begin{abstract}
Electroneurophysiological study
Electroneurophysiological methods play an important role in the study of radiculopathies, helping neurologists and orthopaedic specialists in defining the diagnosis of peripheral deficits by identifying the site, quantifying the extent of the lesion and allowing a follow-up of its evolution. Moreover such methods are the key in performing a differential diagnosis with respect to other peripheral neuropathies, including entrapment neuropathy and plexus neuropathies. However, their implementation must always be guided by careful objective examination.
\end{abstract}

\section{Introduzione}

Lelettroneurofisiologia riveste un ruolo importante nello studio della patologia della colonna vertebrale, $\mathrm{e}$ in particolare nelle radicolopatie, in quanto, pur essendo complementare allo studio clinico, contribuisce alla definizione diagnostica, quantifica un eventuale danno periferico, aiuta a decidere l'opportunità dell'intervento, permette di seguire il decorso e l'evoluzione clinica.

Di fatto, l'esame elettromiografico (EMG) viene richiesto, per una buona percentuale di casi, proprio per il sospetto di una radicolopatia.

\section{Cause di radicolopatie}

Le radici nervose possono essere compresse da varie patologie: ernia del disco;

\section{Editoriale}

validità, ma vengono proposte in maniera non corretta, non rispettando le giuste indicazioni e i limiti, solo per il fatto che "non necessita di ricovero, non serve l'anestesia, si fa soltanto un piccolo tagliettino sulla pelle" e così via, a volte sottacendo le reali conseguenze di gesti non sempre esenti da complicanz e da conseguenze nel tempo, facendo leva sullo stato emozionale de pazienti più che sulla reale validita delle tecniche, forse ignorando che i risultati clinici non sono tanto influenzati dalle tecniche quanto dalle corrette indicazioni e selezion dei pazienti. continua da pag. 1

osteofiti e artrosi; spondilolistesi;

canale vertebrale ristretto;

fratture vertebrali

talvolta tumori extra-midollari.

\section{Quadro clinico della spondi-} loartrosi e dell'ernia del disco

Le cause più frequenti di una radicolopatia sono l'ernia del disco e la spondiloartrosi.

Lernia discale intervertebrale produce una compressione delle radici a un livello completamene endorachideo.

Lartrosi (processo degenerativo di segmenti ossei e articolazioni) della colonna vertebrale viene definita spondiloartrosi e colpisce in modo particolare la colonna cervicale (soprattutto la quinta, la sesta e la settima vertebra) e lombo-sacrale (particolarmente la quarta, la quinta e la prima vertebra sacrale)

Voglio infine ringraziare tutti coloro che hanno partecipato alla stesura di questo numero di Archivio per l'impegno profuso e il tempo sottratto ai loro affetti e alla loro precipua attività; penso che, se chi leggerà questa rivista potrà in qualche modo trovare spunti per un arricchimento culturale o motivi di riflessione, questo sarà per noi motivo di gratificazione e potremo ritenerci ripagati dello sforzo compiuto.

Sergio Brambilla U.O. $4^{a}$ Divisione, Istituto Ortopedico G. Pini, Milano
L'osteoartrosi vertebrale, provocando una compressione della livello della fuoriuscita del forame di coniugazione, determina una sofferenza della radice stessa. La maggioranza dei muscoli del tronco e degli arti riceve conradici e pertanto una lesione monoradicolare difficilmente porterà a un deficit clinicamente eclatante, con atrofia muscolare. La patologia compressiva della colonna vertebrale a carico delle radici nervose di solito interessa sia la componente motoria sia quella sensitiva, e pertanto si avranno sintomi motori e sensitivi. La distribuzione dei sintom e del deficit neurologico rapportata ai vari segmenti permetterà di individuare la sede e di localizzare la lesione.

I sintomi principali legati alla compressione delle radici sono rappresentati da ipostenia, ipotrofia, fascicolazioni, disturb sensitivi e riduzione/assenza de riflessi osteo-tendinei.

\section{Richiami di elettroneuro-} miografia

Lo studio elettroneurofisiologico si compone di 2 parti: elettroneurografia ed elettromiografia. L'elettroneurografia studia la componente motoria e la componente sensitiva dei tronchi nervosi; i suoi principali parametri sono rappresentati da:

studio della velocità di conduzione nervosa $(\mathrm{VdC})$ sensitiva e motoria (Figg. 1, 2);

latenza distale, ampiezza durata e morfologia del potenziale motorio composto (cMAP) o sensitivo (SAP)

Inoltre, essa comprende anche lo studio delle risposte tardive, come l'onda $\mathrm{F}$ e il riflesso $\mathrm{H}$.

Lo studio elettromiografico (EMG) propriamente detto, eseguito con ago coassiale, studia le strutture muscolari. Esso ci da informazioni sul muscolo e indirettamente sulla component radice nervosa, in particolare a temporaneamente fibre da 2 o 3

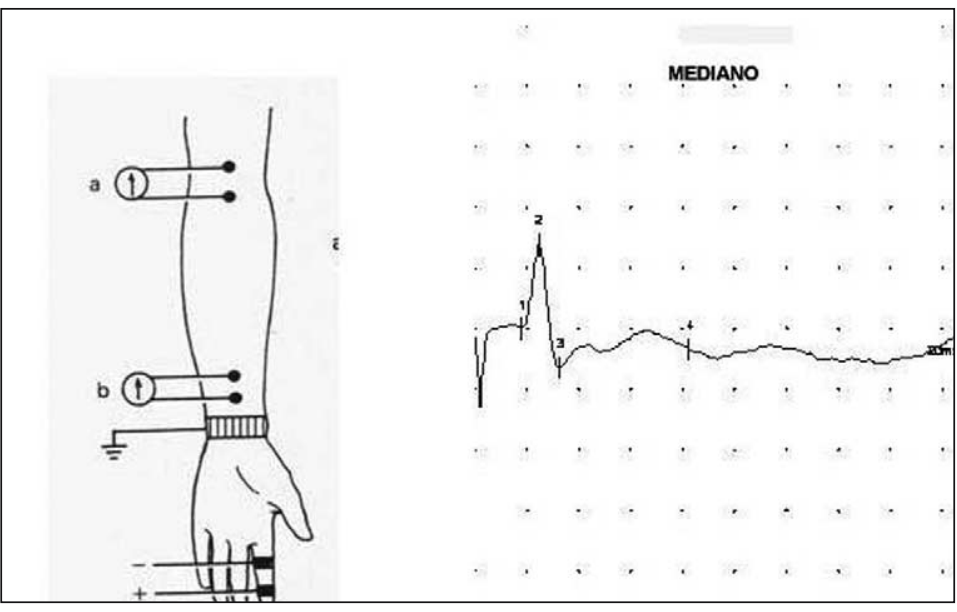

Fig. 2. Potenziale sensitivo (SAP) del nervo mediano Con elettrodi ad anello si stimola in via ortodromica dal secondo dito della mano e si registra con elettrodi di superficie al polso.ll potenziale derivato ha una forma trifasica

nervosa da cui il muscolo dipende

Per quanto riguarda le radicolopatie, l'elettromiografia ci aiuta definire la localizzazione della lesione e talvolta la natura della lesione stessa.

E da tenere presente che, pur esistendo variazioni anatomiche nell'innervazione segmentaria di alcuni muscoli, tuttavia è possibile tracciare una specie di mappa della comune distribuzione radicolare dei muscoli che vengono esaminati (Tabelle 1, 2).

\section{Note di istopatologia}

Le lesioni istopatologiche a carico del nervo sono fondamentalmente di 3 tipi: neuroaprassia assonotmesi e neurotmesi.

La neuroaprassia è la temporanea e parziale lesione della guaina mielinica con conservazion dell'assone o delle strutture periassonali. Tale lesione è reversibile (in genere in qualche settimana) e ha un corrispettivo eletroneurografico, di riduzione della velocità di conduzione nervosa.

Lassonotmesi è data dalla sofferenza dell'assone, con perdita di continuità e degenerazione delle fibre a livello distale, ma con conservazione della mielina, del

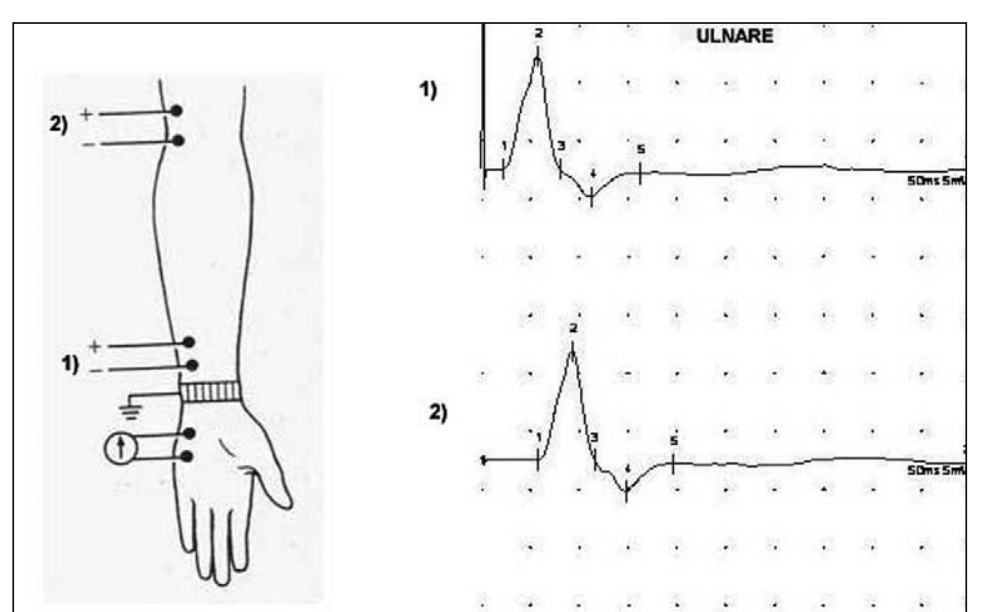

Fig. 1. Misurazione della velocità di conduzione motoria del nervo ulnare. Si deriva con elettrodo di superficie dal muscolo abduttore del quinto dito e si stimola in 2 punti al polso distalmente e al gomito prossimalmente. In questo modo si calcola la velocità di conduzione nervosa motoria dividendo la distanza tra i 2 punti di stimolazione per la differenza delle latenze:VCMM $=\frac{d}{\text { Lat p-Lat } d}=\frac{m}{\mathrm{sec}}$ l'involucro esterno del perinervio e dei tubi endoneurali. Il quadro elettroneurografico è caratterizzato soprattutto da una riduzione dell'ampiezza del potenziale motorio e sensitivo, mentre a livello elettromiografico si registra attività di denervazione nelle fasi iniziali (dopo 2-3 settimane); il recupero clinico può essere anche completo (o quasi) nel giro di qualche mese. La neurotmesi si verifica quando c'è l'interruzione completa del tronco nervoso con perdita di continuità delle fibre assonali, delle guaine mieliniche e connettivali. E la più grave lesione livello clinico ed elettromiografico, con perdita di conduzione nervosa a livello della lesione e con recupero clinico parziale o assente.

\section{Diagnosi differenziale}

Agli arti superiori, le lesioni del nervo ulnare al gomito e del nervo mediano al polso presentano sintomi, come dolore, pare stesie e ipostenia. Tale sintomatologia entra in diagnosi differenziale rispettivamente con la radicolopatia C8/T1 e C6/C7. Così, agli arti inferiori, un deficit del nervo peroneo e del nervo tibiale dovrà essere differenziato da una radicolopatia rispettivamente L5 e S1, in quanto queste due patologie presentano aspetti clinici similari.

Pertanto l'elettroneurofisiologia, associata alla clinica, è decisiva nella diagnosi differenziale delle diverse patologie che possono colpire le strutture nervose periferiche, che vanno dalla radicolopatia, alla plessopatia e alle neuropatie, in specie da intrappolamento.

\section{Quadro EMG nelle radicolopatie}

Una compressione delle radici nervose spinali può coinvolgere sia le fibre sensitive che le fibre motorie, provocando un danno assonale o demielinizzante. 